\title{
A Context-Driven Approach to Route Planning
}

\author{
Hissam Tawfik, Atulya Nagar, and Obinna Anya \\ Intelligent and Distributed Systems Lab, Deanery of Business and Computer Sciences, \\ Liverpool Hope University, Liverpool, United Kingdom L16 9JD \\ \{tawfikh, nagara, 05008721\}@hope.ac.uk
}

\begin{abstract}
Prototyping urban road network routes improves the accessibility of road layouts and enhances people's use of road networks by providing a framework for the analysis and evaluation of routes based on multiple criteria, such as spatial quality, transportation cost and aesthetics. However features identified in this way do not, often, incorporate information about current road condition in order to proactively provide real-time contextual information to users. Context-aware computing has the potential to provide useful information about current road condition by leveraging on contextual information of people, places and things. A prototype, which uses context information and a range of design and user-related criteria to analyse the accessibility of road layouts and plan routes, is developed. A case study is used to validate the prototype.
\end{abstract}

Keywords: road networks, route planning, context-awareness, multi-criteria, simulation.

\section{Introduction}

Space layout and route planning presents a critical challenge as a result of increasing levels of urbanisation and road traffic. Urban planners have always aimed at optimizing the road network design to meet transportation cost, safety, land use, aesthetic and environmental considerations. With the rapid growth in traffic patterns and space utilisation, there is a growing need for a tool to design and evaluate urban road networks based on a number of context-driven criteria. This can be accomplished by efficient computer simulation techniques to aid the analysis of routes based on relevant contextual information about a road network as well as various design and userrelated objectives.

Multi-criteria analysis allows the use of various factors in a route planning scenario. A relevant definition of multi-criteria analysis by [1], views it as a decision-aid and a mathematical tool that allows the comparison of different alternatives or scenarios according to many criteria, often contradictory, in order to guide toward a 'good' decision. The decision maker has to choose from several options or alternatives and will generally have to be contented with a compromising solution. Context-awareness leverages on contextual information of people, places and things to provide useful services necessary to enable real-time determination of optimum route based on multiple objectives. The difficulty in incorporating context-awareness information in route planning arises from the fact that data models for supporting context information are not well-suited for managing geographic information such as proximity 
relationships and; similarly, data models supported by current GIS (Geographic Information Systems) are ill-suited for representing context information [3].

This research work aims to investigate the application of computer modelling and graphical simulation techniques for supporting the evaluation of routes in road networks from a range of design and user-related perspectives, and present a contextdriven approach to route planning. In an earlier part of this work [4], which focused on road network analysis of Liverpool City, it was obtained that a multi-criteria based analysis of urban road network and spatial layouts enables local and global accessibilities of the road network for the set criteria, and the finding of optimum path between two points in a network.

\section{Related Work}

Route planning has been performed using different technologies and algorithms, one of which is Space Syntax [2], a set of analytical and computational tools for the analysis of urban systems. Space syntax can be considered a form of study of geometrical accessibility where a functional relationship is made, or attempted, between the structure of a city and the social, economical and environmental dimensions. A number of space syntax empirical studies have been devoted to the exploration of the complexity and configuration of urban space by searching for a computational representation of a form of geometric order [5]. Space syntax has been successfully applied to relationship between the structure of a city and criminality, way-finding and pollution [6], [7]. According to the space syntax concepts, the accessibility of the spatial layouts is based on the idea that some places or streets are more accessible than others, though the terms used for accessibility, e.g., proximity, integration, connectivity, cost or centrality differ [8].

The concept of network analysis uses Dijkstra algorithm [9] to visualize the network pattern of street structure. Space syntax's Axial Map was applied to evaluate syntactic intelligibility and the degree of natural movement at both towns. Nophaket and Fujii [10] proposed graph geometry for detecting the minimum path structures. However, most researchers argue that space syntax cannot be generalized across different cultures, archetypes and scales of street pattern. Cheverst et al. [11] presented the GUIDE system, a context-aware tourist guide, which provides information about a city to a tourist based on the visitor's profile and contextual information, and is relevant to route planning. Lee and Lee [12] implemented inexact strategy and planning in route planning in Taipei City. Chakhar and Martel [13] presented a strategy for integrating GIS and multi-criteria analysis, and also proposed a design for a spatial decision support system. However, very little research has been carried to formulate prototypes to aid route planning based on the contextual information of a road network.

\section{Road Network Analysis and Route Planning}

We designed a graphical simulation prototype to support multi-objective route evaluation of traffic networks. Our prototype uses a graphical user interface system to enable a user to load, initialise and modify an existing road traffic layout. It then determines 
the best route between two points on the network designated as the start and destination points, based on a combination of distance, safety, road context, comfort and view criteria. Ultimately it allows for the evaluation of the level of accessibility of the local and global road network. The prototype consists of three layers: Visualisation Layer, Road Layout Generation Layer and Road Layout Evaluation Layer, which are described below.

- Visualisation Layer: This layer is the interface between the user and the system where the user inputs the requirements which consist of loading the map, setting the weights for the criteria and the starting and destination points for a given path.

- $\quad$ Road Layout Generation Layer: This layer converts the road map into the connected graph by using junctions as nodes and roads as links, and stores information about distance, safety, comfort and view for all the roads in the network.

- $\quad$ Road Layout Evaluation Layer: This layer is used to obtain the optimum paths in the road network. It uses Dijkstra algorithm [9] to obtain optimum accessibility of the road networks. This layer uses the data obtained from the road layout generation layer and cost function to evaluate accessibility based on multiobjective criteria. The outcome of the multi-criteria analysis is represented visually in this layer, and is used to analyse the local and global accessibility of the road network. Local accessibility of a given road or path can be determined by calculating the mean value of the cost of all roads in the network from the given road, while global accessibility of the whole road network is a result of the average values of the local accessibility of all roads in the network.

The application is developed using the Java programming language and uses applets to construct the user interface (the visualization layer). A typical prototyping scenario starts by loading the road map of the location whose road network is to be evaluated into the prototype. The road map is converted into a connected graph using the nodes and links on the map. The junctions are represented as nodes and roads as links. A data file is used to store information for every road in the road map, such as distance between the connecting node pair, safety of the road, comfort, and view. This information is heuristically determined based on information about the number of turns, number of signals, number of accidents or thefts, etc. The attributes of the road, such as safety, comfort and view are provided between the scales ranging from 1 to 10, where 1 represents the best case and 10, the worst. Fig. 1a and fig. 1b show a graphical representation of a road map and the node-arc matrix format in which the data for the map is stored. In this matrix, the five columns represent the heuristically generated values for the start node, the destination node, the journey's distance, level of safety, level of comfort, and view (aesthetics) level, respectively.

The cost function is designed with the objective of determining the cost of the road according to the multi objective criteria. The cost function is applied to all the possible roads to reach the destination from a starting point. The selection of optimum path is based on the cost of path, denoted as $\mathrm{C}_{\mathrm{f}}$.

$$
\mathrm{C}_{\mathrm{f}}=\sum_{i=1}^{n} \mathrm{C}_{\mathrm{i}} * \mathrm{~W}_{\mathrm{i}}
$$

Where $n$ is the number of criteria (safety, comfort, view), $C_{i}$ is the cost of the road for criterion $\mathrm{i}, \mathrm{W}_{\mathrm{i}}$ is the weight of criterion $\mathrm{i}$ provided by the user. 


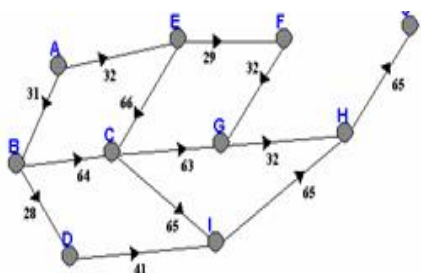

Fig. 1a. Link-node representation of a road map

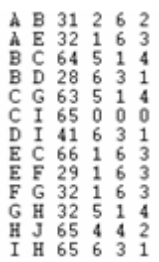

Fig. 1b. Node-arc Matrix

Dijkstra's algorithm [9] is used to choose the optimum path, i.e. the path with minimum cost. Dijkstra's algorithm solves the single source shortest path problem on a weighted, directed graph in which all edge weights are non-negative.

The input to the algorithm consists of a weighted directed graph $\mathrm{G}$, the road network converted to connected graph, which takes the roads as edges and junctions as nodes. Let $\mathrm{s}$ be the source node in $\mathrm{G}$ from where the user wants to travel, and $\mathrm{t}$, the destination point. Let V denote the set of all nodes in graph G. Each edge of the graph is an ordered pair of vertices $(\mathrm{u}, \mathrm{v})$ representing a connection from node $\mathrm{u}$ to node $\mathrm{v}$. The set of all edges is denoted by E. Weights of edges are given by a weight function $\mathrm{w}: \mathrm{E} \rightarrow[0, \infty]$; therefore $\mathrm{w}(\mathrm{u}, \mathrm{v})$ is the non-negative cost of moving from node $\mathrm{u}$ to node $\mathrm{v}$. The cost of an edge is determined by using the cost function. The cost of a path between two nodes is the sum of costs of the edges in that path. For a given pair of nodes $s$ and $t$ in $\mathrm{V}$, the algorithm finds the path from $s$ to $t$ with lowest cost. It is also used for finding costs of minimum cost paths from a single node $s$ to all other nodes in the graph.

Dijkstra's algorithm with directed graph $G=(V, E)$, maintains a set $S$ of vertices whose final shortest path weights from the source $s$ have already been determined. The algorithm repeatedly selects the node $u \in V-S$ with the minimum cost path estimate, adds $u$ to $S$ and relaxes all edges leaving $u$. Hence an optimum path is determined by calculating the cost of every road using cost function and finding the path with lowest cost using Dijkstras algorithm.

\section{Role of Context-Awareness in Route Planning}

Context is defined as any measurable and relevant information that can be used to characterise the situation of an entity, such as a road network route [14]. Techniques in context-awareness computing employ temporal context information to proactively inform a user of relevant information about current road condition so as to guide them in making decisions about the best route to take for a particular trip. Context is highly dynamic in space and time [15], and as such can be used to provide real-time information about the condition of a road network route and increase the probability of a road user selecting the optimum route to a destination.

The use of context-awareness in our simulation prototype enables us to build a route planning prototype that improves the local and global accessibility of the road network, and allows users to consider current road condition in making decisions about the route to take by: (1) assessing the situation, (2) identifying the available options, (3) determining the costs and benefits of each option, and (4) selecting the 
option with the lowest and highest benefits [16]. More specifically, the use of context services enables a road user to extract and model:

- Information reflecting current road context, which can be used to react to current events, such as "it is raining", "road repair work going on $5 \mathrm{~km}$ ahead, there is likelihood of delay", or "high traffic density expected."

- Critical safety information, such as "an accident just occurred $2 \mathrm{~km}$ ahead beside Albert Dock."

- Information relating to the wider environmental context, such as "route $\mathrm{C}$ is the most aesthetic route to your destination, and has the following attractions: St Johns park and front view Liverpool Cathedral."

- Information about a business interest or a particular user interest, such as "Hotel A situated $3 \mathrm{~km}$ ahead has available rooms.

\section{Route Planning and Prototyping Scenario}

In [4], a road network within a construction site was used to demonstrate our approach. In this work, we incorporate context as a criteria, and illustrate this using Liverpool city centre road network. The road network layouts were analysed in terms of their accessibility based on different criteria as well as the prevailing road context. To achieve this, weights are assigned to each of the criteria, such as distance, safety, comfort and aesthetics. Based on these criteria the following are obtained: (1) Local accessibility of the road network for the set criterion, (2) Global accessibility of the road network and, (3) Optimum path between two points based on multi-criteria.

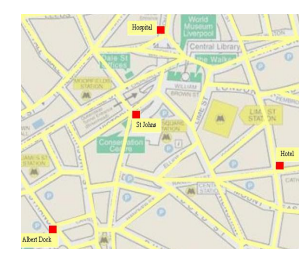

Fig. 2a. Liverpool city centre map

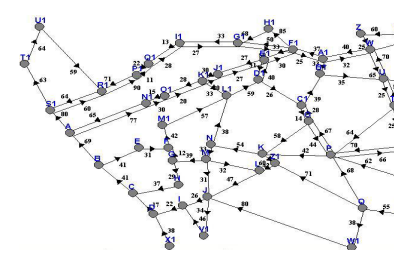

Fig. 2b. Link-node Graph for Liverpool city centre road network

Various scenarios are described to demonstrate the use of the prototype. The road network map of Liverpool city centre is converted into a connected graph using the nodes and links on the map. The junctions are represented as nodes and roads as links.

Fig. 2a shows the map of Liverpool city centre, and fig. 2b shows the corresponding connectivity graph. The values for the criteria are predefined for different roads. See fig. 1. The map highlights four locations - Hotel, the Albert Dock, St Johns and the Royal Hospital - chosen to illustrate context-driven route planning. There is more than one path from a source location to chosen destination location. The optimum path obtained by the network analysis is based on the criteria and weights specified. Using equal weights for the various criteria - distance, safety, traffic density, view 


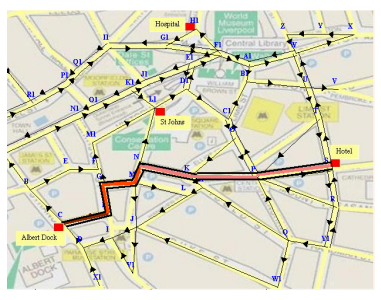

Fig. 3a. Path from Hotel to Albert Dock with equal weights

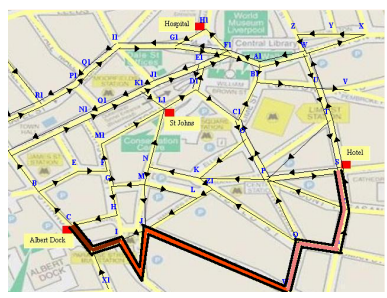

Fig. 4a. Path from Hotel to Albert Dock with high aesthetic appeal

Low accessibility

High accessibility

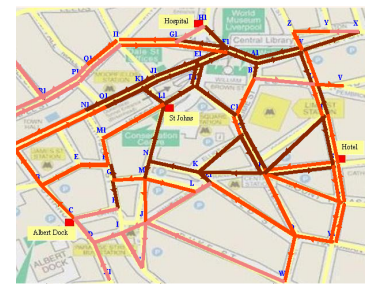

Fig. 3c. Global accessibility for multi criteria analysis with equal weights

\section{Low accessibility \\ High accessibility}

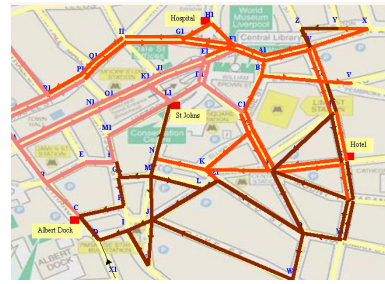

Fig. 3b. Local accessibility for multi criteria analysis with equal weights

\section{Low accessibility High accessibility}

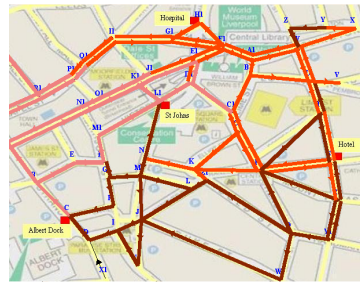

Fig. 4b. Local accessibility of Albert Dock with high aesthetic appeal

Low accessibility ___ High accessibility

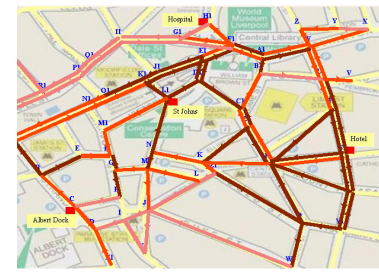

Fig. 4c. Global accessibility of Albert Dock with high aesthetic appeal

and comfort, a context-driven analysis for a path from Hotel to Albert Dock is obtained. Fig. 3a to fig. 3c show the optimum path, local accessibility and global accessibility using equal weights.

In the second scenario the same set of multi criteria - distance, safety, view and comfort are assigned different weights to account for preferences. To analyse the road network based on the high aesthetic appeal from Hotel to Alert Dock, the weight for view criteria is set to high. The optimum path after analysis is shown in fig. 4a to fig. 4c. The local accessibility and global accessibility based on high aesthetic appeal are indicated in fig. $4 \mathrm{~b}$ and fig. $4 \mathrm{c}$. In the third scenario, contextual information represented by the current road condition is used as a criterion. The contextual data 
employed is the varying density of traffic on the route from Hotel to Alert Dock, which is assigned different weights based on the time of the journey. To analyse the road network based on the current road condition from Hotel to Alert Dock, the weight for high traffic density is set to high.

The case study demonstrates the concept of accommodating for more than one aspect of road context, considered as criteria, in route planning. The study considered the quality and aesthetics of the journey, current road condition and levels of safety and comfort in addition to the conventional travel time, distance and cost. The results demonstrate that for the path chosen from source to destination, local accessibility and global accessibility will vary based on the weights assigned to the criteria. The criteria or contextual information emphasised depends on the type of user. For example, new visitors to a city would typically like to take the safest route to their destination. Tourists, generally, are more interested in aesthetics, and would go through routes that present the most beautiful views of a city. Habitual road users are interested in saving time and cost as well as comfort, and would always take the shortest routes and those with less number of turns and lower traffic density. Emergency services providers, such as ambulance drivers and the police are more likely to prioritise short routes over other criteria.

\section{Conclusion}

This work investigates the application of computer simulation and modeling techniques to the analysis, evaluation and optimization of road networks based on context information about the road network. Our prototype uses multi-criteria evaluation to determine the optimum path based on current road condition, such as traffic density, and a range of multiple criteria, such as distance, safety, comfort and view. A contextdriven prototype could potentially support route planners in early evaluation of road network layout in terms of its time, cost, road context and safety implications on road users and pedestrians, and to assist with the provision of enhanced road network layouts. Our future work will focus on research to accommodate for complex road scenarios and traffic restrictions.

Acknowledgement. The authors would like to thank S. Dasari for the contribution to the programming phase of this work.

\section{Reference}

1. Roy, B.: Multicriteria Methodology for Decision Analysis. Kluwer, Dordrecht (1996)

2. Hillier, B.: Space is the machine. Cambridge University Press, Cambridge (1996)

3. Coyle, M., Shekhar, S., Liu, D., Sarkar, S.: Experiences with Object Data Models in Geographic Information Systems. Internal Technical Report, Department of Computer Science, University of Minnesota, US (1997)

4. Soltani, A.R., Tawfik, H., Fernando, T.: A multi-criteria based path-finding application for construction site layouts. In: Proc. of 6th Int'l Conf on Information Visualisation, London, UK (July 2002)

5. Jiang, B., Claramunt, C.: Topological Analysis of Urban Street Networks. Environment, Planning \& Design 31(1), 151-162 (2003) 
6. Peponis, J., Zimring, C., Choi, Y.: Finding the building in way finding. Environment and Behavior 22, 555-590 (1990)

7. Ortega, A., Jiménez, E., Jiménez, C., Mercado, S., Estrada, C.: Sintaxis espacial: una herramienta para la evaluación de escenarios. XV meeting of the Mexican Society of Behavioral Analysis, México (2001)

8. Porta, S., Crucitti, P., Latora, V.: The Network Analysis of Urban Streets: A Primal Approach. Environment and Planning B: Planning and Design 33(5), 705-725 (2005)

9. Dijkstra, E.W.: A note on two problems in connection with graphs. Numerische Mathematik 1, 269-271 (1959)

10. Nophaket, N., Fujii, A.: Syntactic and Network Pattern Structures of City - Comparison of Grid and Meandering Street Patterns in Kyojima and Honjo. Journal of Asian Architecture and Building Engineering, JAABE 3(2), 349-356 (2004)

11. Cheverst, K., Davies, N., Mitchell, K., Friday, A.: Experiences of Developing and Deploying a Context-Aware Tourist Guide: The GUIDE Project. In: Proc of MOBICOM. ACM Press, Boston (2000)

12. Lee, H., Lee, C.: Inexact strategy and planning-the implementation of route planning in Taipei city. In: Proc. of the 1996 Asian Soft Computing in Intelligent Systems and Information Processing, Kenting, Taiwan, vol. 1996, pp. 308-313 (1996)

13. Chakhar, S., Martel, J.: Enhancing Geographical Information Systems Capabilities with Multi-Criteria Evaluation Functions. Journal of Geographic Information and Decision Analysis 7(2) (2003)

14. Dey, A., Abowd, G.: Towards a better understanding of context and context-awareness. In: Conference on Human Factors in Computing Systems (CHI 2000): Workshop on the What, Who, Where, When and How of Context-Awareness, The Hague (April 2000)

15. Rakotonirainy, A.: Design of context-aware systems for vehicles using complex system paradigms. In: de Lavalette, B.C., Tijus, C. (eds.) Proc. of the CONTEXT 2005 Workshop on Safety and Context, Paris, France (July 2005)

16. Strauch, B.: Investigating human error: Incidents, accidents and complex systems, Ashgate Publishing Limited (2002) 